\title{
Severe delayed posttonsillectomy haemorrhage due to a pseudoaneurysm of the lingual artery
}

\author{
Nynke van Cruijsen · Joost Gravendeel • \\ Frederik G Dikkers
}

Received: 12 December 2006 / Accepted: 22 June 2007 / Published online: 17 July 2007

(C) Springer-Verlag 2007

\begin{abstract}
A 3.5-year-old child is presented with severe, recurrent haemorrhages after a tonsillectomy. The haemorrhages were caused by a pseudoaneurysm of the lingual artery, which was visualised more than 2 weeks postoperatively by angiography. The pseudoaneurysm was successfully embolised by coils. Coiling is an adequate therapeutic option for severe posttonsillectomy haemorrhage due to arterial damage.
\end{abstract}

Keywords Pseudoaneurysm · Lingual artery · Posttonsillectomy haemorrhage $\cdot$ Embolisation

\section{Introduction}

Postoperative haemorrhage is the most common serious complication of adenotonsillectomy and its incidence is around 3\% [1]. Intraoperative bleeding $(<24 \mathrm{~h})$ may be related to the surgical technique or a bleeding diathesis. Delayed postoperative haemorrhage $(>24 \mathrm{~h}$ ) predominantly occurs on the fifth to seventh-day postoperatively when the slough separates from the granulating fossa. The most critical posttonsillectomy haemorrhages are due to arterial dissections and aneurysms. In this report we present a boy

N. van Cruijsen $(\varangle) \cdot$ F. G Dikkers

Department of Otorhinolaryngology,

University Medical Center Groningen,

University of Groningen, P.O. Box 30.001,

9700 RB Groningen, The Netherlands

e-mail: n.van.cruijsen@kno.umcg.nl

J. Gravendeel

Department of Radiology,

University Medical Center Groningen,

University of Groningen, Groningen, The Netherlands with a severe, delayed posttonsillectomy haemorrhage due to a pseudoaneurysm of the lingual artery.

\section{Case report}

An otherwise healthy 3.5-year-old boy was brought in with severe oral bleeding. Nine days prior to the presentation he had undergone an adenotonsillectomy for chronic adenotonsillitis in another hospital. The tonsils had been removed with cold dissection and sutures had been placed in both tonsil areas. The procedure and the immediate postoperative period went uncomplicated. On the fifth postoperative day he had been admitted for a one-night observation because of haemorrhage from the mouth. During the admission no active bleeding was observed and his haemoglobin level of $4.9 \mathrm{mmol} / \mathrm{l}$ (normal values: $6.5-8.4 \mathrm{mmol} / \mathrm{l}$ ) was treated conservatively.

The second postoperative bleeding started 9 days postoperatively, late at night when the patient was lying in bed. There were no provoking events. The bleeding lasted around $10 \mathrm{~min}$ but had already stopped at arrival in our emergency room. We saw a pale and restless boy. Intra-oral examination in general anaesthesia showed lacerated pharyngeal muscles in the left tonsillar fossa and a loose suture was removed. The right tonsillar fossa revealed diffuse bleeding from the pharyngeal muscles without pulsating masses. The nasopharynx and tongue base showed no abnormalities. The haemoglobin was increased from 2.9 to $8.2 \mathrm{mmol} / \mathrm{l}$ by transfusion with two units of packed red blood cells. The coagulation values were within the normal range. The postoperative period was uncomplicated, so he was discharged after 5 days, 15 days after the primary intervention, with dietary advices.

One day later the boy arrived by ambulance with heavy bleeding from the mouth. He was pale, shivering and 
drowsy. Again, the severe bleeding started without a provoking moment and persisted for about $15 \mathrm{~min}$. Immediately, after arrival at our emergency department, he was re-explored intra-orally under general anaesthetics. We saw a bluish, pulsating mass of 5 by $12 \mathrm{~mm}$ low in the right tonsillar fossa without active bleeding. The faucial pillars on the right side were approximated without tearing the mucosa or induration of the aneurysm. However, during extubation a massive bleeding occurred. The boy was reintubated, and with digital oral pressure during $15 \mathrm{~min}$ haemostasis was achieved.

The patient was stabilised and an arteriography was performed. A pseudoaneurysm of the right lingual artery was visualized with selective catheterisation using a vertebral catheter (Fig. 1). The pseudoaneurysm was coiled distally and proximally using a microcatheter (Excelsior 10, Boston scientific, Natick, MA, USA) and microcoils (GDC 10, Boston Scientific, Natick, MA, USA). The close relation of the proximal part of the pseudoaneurysm to the entrance of the facial artery made coiling of the facial artery inevitable. The pseudoaneurysm was coiled successfully and no bleeding focus was seen on the control arteriography (Figs. 2, 3). During the procedures blood transfusion was needed to restore the low haemoglobin level.

The boy was extubated without any bleeding and 4 days after the embolisation he was discharged in a good clinical condition. At a follow-up examination, he did not report any intra-oral or neurological abnormalities.

\section{Discussion}

True aneurysms develop through congenital or acquired weakness of all three layers of the vascular wall. False or



Fig. 1 Pseudoaneurysm of the right lingual artery seen from rightanterior side (3D-surface rendering)

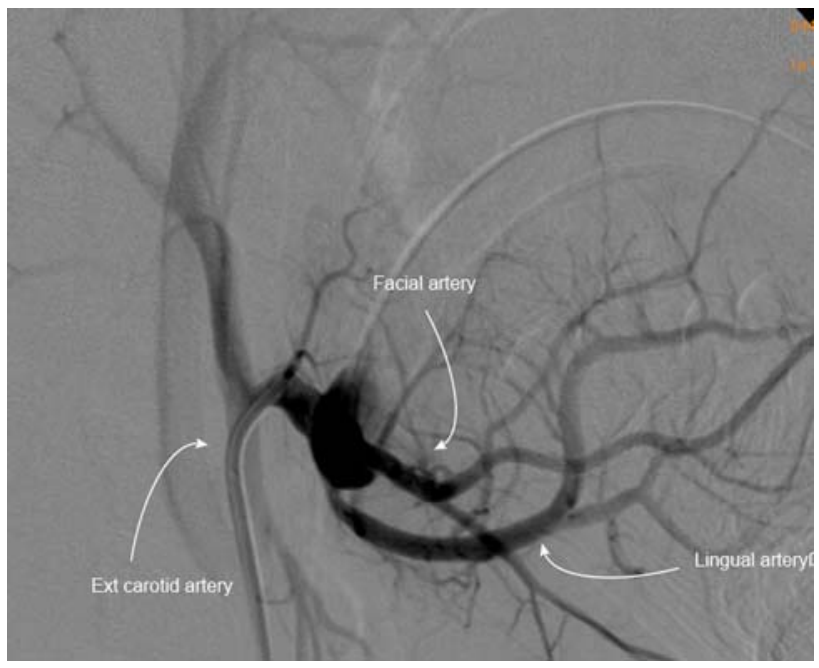

Fig. 2 Arteriography of pseudoaneursym of the right lingual artery seen from right-anterior side

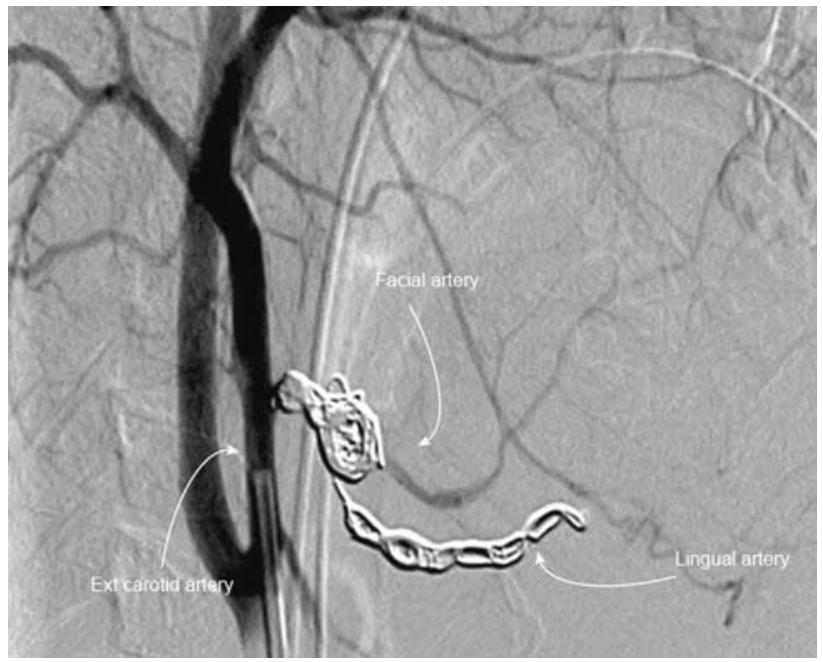

Fig. 3 After coiling of the pseudoaneurysm, including the facial artery; compare to Fig. 2

pseudoaneurysms may arise due to localised arterial wall laceration caused by blunt or penetrating trauma. The intima or adventitia layer of the vessel wall is dissected, which creates a periarterial haematoma. Pseudoaneurysms after tonsillectomy may be triggered by blunt or direct trauma during dissection or due to placing ligation sutures.

Haemorrhages from posttonsillectomy pseudoaneurysms of the lingual, facial and internal carotid artery have been observed within $5 \mathrm{~h}$ postoperatively to the eighth postoperative day in a few cases [2, 3]. In those reports no intra-oral pulsating masses were observed. The pseudoaneurysms were shown with angiography and treated with embolisation. Mitchell et al. reported a case of fulminant bleeding during the tonsillectomy procedure from an aberrant lingual artery with a pseudoaneurysm, which was treated with embolisation [4]. A pulsating neck mass can also be the 
first symptom of a pseudoaneurysm, which has been seen in two cases without any bleeding [5, 6].

It is striking that the above-mentioned cases of posttonsillectomy pseudoaneurysms concerned children under the age of 10 years. No cases of posttonsillectomy haemorrhage due to pseudoaneurysms in adults have been reported. This suggestive higher incidence of pseudoaneurysms in children might result from the smaller anatomy and thinner pharyngeal muscles, and a subsequent higher risk of damaging the large vessels.

Since the angiography of our patient did not show any aberrant courses of arteries, trauma to the artery during dissection or placing the suture in the tonsillar fossa could have caused the pseudoaneurysm in our patient. In the two first oral inspections no masses were observed, only after the third bleeding (16th day postoperatively) a pulsating mass was seen. This suggests a gradual development of the aneurysm. False aneurysms are known to expand when the periarterial clot dissolves and more blood flows into the periarterial space [5]. The size of the lesion to the vessel wall might determine the amount of blood and whether the pseudoaneurysm is large enough to be observed. Maybe the vessel lesion in our patient was small, which made the pseudoaneurysm grow slowly. Although no real provoking events seem to have initiated the bleedings, lying down in bed might have raised the blood pressure in the carotid artery, which made the aneurysm leak. A new clot made the severe short lasting bleeding stop.

Severe posttonsillectomy haemorrhage is treated with external ligation of the internal or external carotid artery or endovascular coiling of the injured artery. Although a randomized controlled trial has not been performed, embolisation seems to have advantages over external ligation [7]. Firstly, the diagnostic evaluation can be combined with direct therapeutic intervention. Furthermore, coiling is more selective. This is especially convenient in posttonsillectomy haemorrhages, where several branches of the external or internal carotid artery could be damaged. The third advantage is that embolisation is less mutilating and has less risk of damaging the vagal and accessory nerves.
The potential complications of embolisation are perforation of the vessel with leakage of coils, ischemia of mucosa or nerves, or vasospasm. External ligation seems to be the only quick option in massive bleeding and unstable patients.

\section{Conclusion}

Pseudoaneurysms are life-threatening and should be considered in severe posttonsillectomy haemorrhage. Pseudoaneurysms do not always present as intra-oral or cervical pulsating masses, and can develop gradually. If the technique and technical crew are available, interventional arteriography is strongly recommended in severe posttonsillectomy haemorrhage. It is diagnostic and therapeutic at the same time and more selective than surgical ligation.

\section{References}

1. Myssiorek D, Alvi A (1996) Post-tonsillectomy hemorrhage: an assessment of risk factors. Int $\mathrm{J}$ Pediatr Otorhinolaryngol 37(1):35-43

2. Simoni P, Bello JA, Kent B (2001) Pseudoaneurysm of the lingual artery secondary to tonsillectomy treated with selective embolization. Int J Pediatr Otorhinolaryngol 59(2):125-128

3. Weber R, Keerl R, Hendus J, Kahle G (1993) The emergency: traumatic aneurysm in the area of the head-neck. Laryngorhinootologie 72(2):86-90

4. Mitchell RB, Pereira KD, Lazar RH, Long TE, Fournier NF (1997) Pseudoaneurysm of the right lingual artery: an unusual cause of severe hemorrhage during tonsillectomy. Ear Nose Throat $\mathrm{J}$ 76(8):575-576

5. Defatta RJ, Verret DJ, Bauer P (2005) Extracranial internal carotid artery pseudoaneurysm. Int J Pediatr Otorhinolaryngol 69(8):11351139

6. Karas DE, Sawin RS, Sie KC (1997) Pseudoaneursym of the external carotid artery after tonsillectomy. A rare complication. Arch Otolaryngol Head Neck Surg 123(3):345-347

7. Opatowsky MJ, Browne JD, McGuirt JW Jr, Morris PP (2001) Endovascular treatment of hemorrhage after tonsillectomy in children. AJNR Am J Neuroradiol 22(4):713-716 